\title{
An Electron Spin Resonance Method for Determination of Orientation to Two Directions in Polytetrafluoroethylene
}

\author{
Shigetaka SHIMADA, Yasurō HoRI, and Hisatsugu KASHIWABARA \\ Nagoya Institute of Technology, Gokiso-cho, Showa-ku, Nagoya 466, Japan
}

(Received February 28, 1984)

\begin{abstract}
A computer simulation program to calculate the ESR spectrum of peroxy radicals partially oriented in two directions was developed. The calculation was applied to rigid peroxy radicals, $\sim \mathrm{CF}_{2}-\mathrm{CFOO} \cdot-\mathrm{CF}_{2} \sim$, trapped in crystalline regions of elongated polytetrafluoroethylene films with a stretch ratio 3.6. The degree of orientation was estimated as follows; $f_{\alpha}=$ $0.45 \pm 0.02$ and $f_{\alpha^{\prime}}=0.10 \pm 0.02$, where $f_{\alpha}$ and $f_{\alpha^{\prime}}$ are the degree of orientation of polymer chains in the stretching direction and the film plane, respectively.

KEY WORDS ESR / Peroxy Radical / Partial Orientation / Two Direction / Polytetrafluoroethylene /
\end{abstract}

Because most polymeric materials exhibit some anisotropy in their properties, for example, mechanical and dielectric properties, their macroscopic form depend on the fine arrangement of the main chains. Hence, a knowledge of chain orientation distribution is essential for understanding the relation between morphology and bulk properties. One can often induce a preferred orientation of polymer main chains by deformation of polymer samples through stretching or rolling. It is well known that uniaxial stretching produces preferentially uniaxial orientation of cylindrical symmetry, and biaxial stretching and rolling bring about orientation parallel to the plane preferentially. To completely characterize the orientation of polymer chains, two orientation functions,

$$
f_{\alpha}=\left\langle\left(3 \cos ^{2} \alpha-1\right)\right\rangle / 2
$$

and

$$
f_{\alpha^{\prime}}=\left\langle\left\{3 \cos ^{2}\left(\alpha^{\prime}-\pi / 2\right)-1\right\}\right\rangle / 2
$$

must be determined, where $\alpha$ and $\alpha^{\prime}$ are the angles between the polymer chain axis and the stretching direction and between the axis and the film plane, respectively. The orientation functions have been determined for partially crystalline and amorphous polymers by various means such as the X-ray pole figure method, birefringence, electron microscopy and mechanical measurements. ${ }^{1}$ In this report, we suggest a simple ESR method to study the angular dependence of the ESR spectrum obtained from a spin label incorporated in a partially ordered polymer substance. The ESR method offers some distinct advantages for obtaining information on local molecular orientations in polymer samples, including orientations in amorphous and crystalline regions, the inside and end parts of polymer chains, and these in the molecularly disordered regions, by introducing the spin labels into the respective places. A number of workers ${ }^{2-6}$ have studied to anisotropic ESR spectra of peroxy radicals trapped in solid polymers and applied the results obtained to molecular motion and the orientation. As a spin probe, we used a peroxy radical, $\sim \mathrm{CF}_{2}-\mathrm{CFOO} \cdot-\mathrm{CF}_{2} \sim$, in the inside parts of a polytetrafluoroethylene 
chain.

\section{EXPERIMENTAL}

A polytetrafluoroethylene (PTEE) film, AFLON (a product of Asahi Glass Co., Ltd.), in which polymer chains were considerably oriented, was stretched at $373 \mathrm{~K}$ at a stretch ratio 3.6. This elongated film was then cut into narrow strips which were then stacked together and placed in Spectrosil sample tubes. Samples were prepared so that the stretching direction in the film was either perpendicular (case I) or parallel (case II) to the axis of the sample. The sample tubes were then sealed under $10^{-5}$ torr and irradiated at room temperature with ${ }^{60} \mathrm{Co} \gamma$ rays, the total does being ca. 30 Mrad. After verification that the observed ESR spectrum showed mainly the alkyl radicals, $\sim \mathrm{CF}_{2} \dot{\mathrm{C} F C F} F_{2} \sim$, and a small amount of the end radicals, $\sim \mathrm{CF}_{2} \mathrm{CF}_{\dot{ }}$, air at atmospheric pressure was introduced into the sample tubes at room temperature for oxidation.

The ESR spectrum of the peroxy radicals observed at room temperature are composed of two components with anisotropic and isotropic angular dependences on an external magnetic field which arise from peroxy radicals trapped in the crystalline and amorphous regions.

Peaks due to the isotropic component disappeared on introducing ethylene into the sample tube under 500 torr at $328 \mathrm{~K}$ for a long time (more than a week). ${ }^{5}$

The ESR measurements were carried out with an $X$ band spectrometer, JEOL ME3X, operated with $100 \mathrm{kHz}$ modulation. The spectra were recorded by a MELCOM 70/25 computer connected to a spectrometer. The signal of diphenyl-picryl-hydrazyl (DPPH) was used as a standard for $g$-values. The magnetic field sweep was calibrated with a known splitting $\mathrm{Mn}^{2+}$ constant.
Simulation of the ESR Spectrum of Peroxy Radicals Partially Oriented in Two Directions

For an interpretation of ESR spectrum of peroxy radicals, it is necessary to calculate the resonance magnetic field, $H_{\mathrm{r}}(\Omega)$ and intensity of the ESR transition, $I\left(H_{\mathrm{r}}\right)$, for a paramagnetic center with a particular arbitrary orientation with respect to an external magnetic field. The direction of the external field, $\Omega$ represents the set of direction cosines of the external field, $(\cos \theta, \sin \theta \sin \phi, \sin \theta \cos \phi)$, expressed on the principal axis system of the $g$ tensor. Figure 1 represents the geometrical relationships for partially oriented peroxy radicals. Two measurements must be made for the analysis of the angular dependent ESR spectra of peroxy radicals partially oriented in two directions. As shown in Figure 1(A), samples were prepared so that the stretching direction in the film stack (MD) was either perpendicular (case (I)) or parallel (case (II)) to the axis of the sample tube, which in turn is parallel to $O X^{\prime}$ (case (I)) or $O Z^{\prime}$ (case (II)).

The angles of rotation, $\delta$, can be defined as that between the external field, $O H_{0}$ and stretching direction, MD (case (I)). In the same manner, the angle $\delta^{\prime}$ can be defined as that between the external field, $O H_{0}$ and the normal axis to the film plane, ThD (case (II)). For simplicity, we assume one of the principal axes $\left(g_{1}\right)$ to be parallel to the polymer chain axis, as shown in Figure 1(c). This assumption holds for the case of PTFE as concluded by Iwasaki and Sakai. ${ }^{6}$ The resonance field can be easily calculated as follows.

$$
\begin{aligned}
H_{\mathrm{r}}(\theta, \phi)= & h v / g(\theta, \phi) \beta \\
g^{2}(\theta, \phi)= & g_{1}{ }^{2} \cos ^{2} \theta+g_{2}{ }^{2} \sin ^{2} \theta \sin ^{2} \phi \\
& +g_{3}{ }^{2} \sin ^{2} \theta \cos ^{2} \phi
\end{aligned}
$$

where $h v$ and $\beta$ are the microwave energy and Bohr magneton, respectively. The intensity of the resonance field, $I\left(H_{\mathrm{r}}\right)$, is proportional to the number of peroxy radicals, in $\theta, Q(\theta)$. The function $Q(\theta)$ depends on the product, $P_{\mathrm{M}}(\alpha)$. 
(A) Sample Axis System

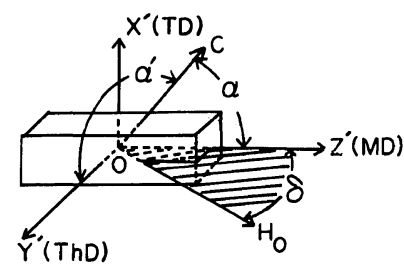

(B) Principal Axis System

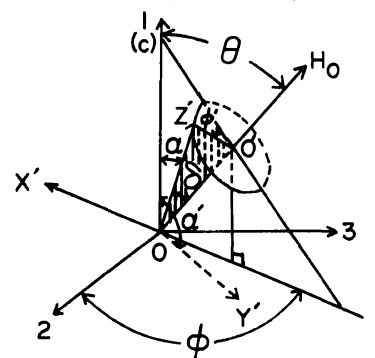

(a) - Case (I)
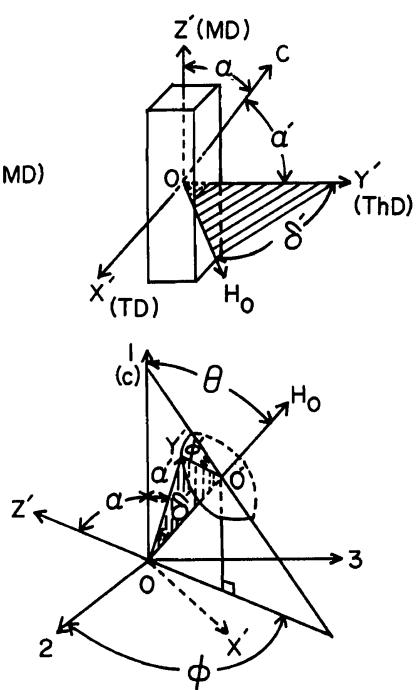

(b) Cose (II)

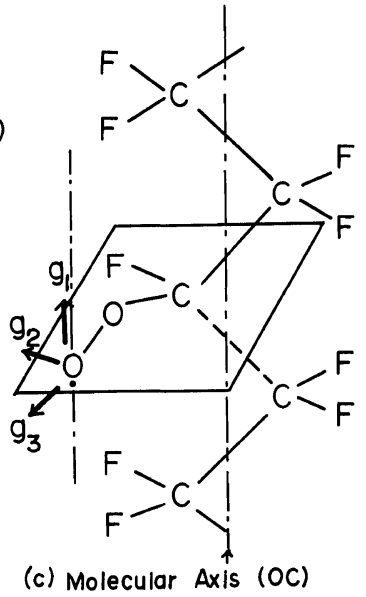

(c) Molecular Axis (OC)

Figure 1. Two different axis systems and polymer chain (OC). $Z^{\prime}$ (MD) represents the machine direction (stretching direction), $X^{\prime}$ (TD) the transverse direction and $Y^{\prime}$ (ThD), the direction perpendicular to the surface of the sheet. (a)-Case I: an external magnetic field $\left(O H_{0}\right)$ rotated on the surface of the sheet, $Y^{\prime} Z^{\prime}$. $O Y^{\prime}$ is in the plane $O^{\prime} O Z^{\prime}$. (b)-Case II: an external magnetic field $\left(O H_{0}\right)$ rotated in the plane perpendicular to the machine direction, $X^{\prime} Y^{\prime} . O X^{\prime}$ is in the plane, $O^{\prime} O Y$. (c): Schematic representation of PTFE peroxy radicals.

$P_{\mathrm{Th}}\left(\alpha^{\prime}\right)$. The functions $P_{\mathrm{M}}(\alpha)$ and $P_{\mathrm{Th}}\left(\alpha^{\prime}\right)$ are distribution functions of the polymer chains with respect to the reference axes, MD and $\mathrm{ThD}$, respectively. The functions are assumed to be Boltzman distributions. ${ }^{7)}$

$$
\begin{aligned}
& P_{\mathrm{M}}(\alpha)=\exp \left(a \sin ^{2} \alpha\right) \\
& P_{\mathrm{Th}}\left(\alpha^{\prime}\right)=\exp \left(a^{\prime} \sin ^{2}\left(\alpha^{\prime}-\pi / 2\right)\right)
\end{aligned}
$$

The angles $\alpha$ and $\alpha^{\prime}$ can be calculated for a particular arbitrary orientation of the peroxy radical and an arbitrary rotation angle, which are represented as $\theta$ and $\delta$ (or $\delta^{\prime}$ ), respectively. All orientations of the sets of the external field $O H_{0}$ and the reference axis $O Z^{\prime}$ (case (I)) (or $O Y^{\prime}$ (case (II)) for constant angle, $\delta$, can be represented on the principal axis system by changing the angles, $\theta, \phi$ and $\phi^{\prime}$ from $O$ to $2 \pi$, as shown in Figure 1. Here, the angle $\phi^{\prime}$ is the dihedral angle between the planes, $O O^{\prime} 1$ and $O O^{\prime} Z^{\prime}$ (case (I)) (or $O O^{\prime} Y$ (case (II)). When the external field is rotated in the film plane $\left(Y^{\prime} Z^{\prime}\right)$ (case (I)),

$$
\begin{aligned}
& \cos \alpha=\cos \theta \cos \delta+\sin \theta \sin \delta \cos \phi^{\prime} \\
& \cos \alpha^{\prime}=\cos \theta \sin \delta-\sin \theta \cos \delta \cos \phi^{\prime}
\end{aligned}
$$

When the external field is rotated in the plane perpendicular to the direction of stretching $\left(X^{\prime} Y^{\prime}\right)$ (case (II))

$$
\begin{aligned}
& \cos \alpha=\sin \theta \sin \phi^{\prime} \\
& \cos \alpha^{\prime}=\cos \theta \cos \delta^{\prime}+\sin \theta \sin \delta^{\prime} \cos \phi^{\prime}
\end{aligned}
$$

The total probability distribution function of the molecular axis, $Q(\theta)$, for the fixed orientation $(\theta, \phi)$ should be represented by the following equation.

$$
Q(\theta)=\int_{0}^{2 \pi} P_{\mathrm{M}}(\alpha) \cdot P_{\mathrm{Th}}\left(\alpha^{\prime}\right) \mathrm{d} \phi^{\prime}
$$

The function, $Q(\theta)$ is independent of the angle $\phi$. 
For determination of the orientation functions $f_{\alpha}$ and $f_{\alpha^{\prime}}$, a computer simulation was made in the following way:

(a) The distribution functions $P_{\mathrm{M}}(\alpha)$ and $P_{\mathrm{Th}}\left(\alpha^{\prime}\right)$ were calculated for all angles of $\alpha$ and $\alpha^{\prime}$ and the results stored in the computer using the given parameters $a$ and $a^{\prime}$ (eq 2).

(b) For a given angle of rotation, $\delta$ or $\delta^{\prime}$, the angles $\alpha$ and $\alpha^{\prime}$ were calculated (eq 3 or 4 ) and $Q(\theta)$ then (eq 5) for all angles $\theta$.

The results were stored in the computer. (c) Using the principal values, $g_{1}=2.0028$, $g_{2}=2.0073$, and $g_{3}=2.0385$ obtained from simulation of the ESR spectra of powdered PTFE sample, the resonance field $H_{\mathrm{r}}$ and the intensity were calculated.

$$
\begin{aligned}
I(H)= & \int_{0}^{2 \pi} \int_{0}^{2 \pi} Q(\theta) G\{H \\
& \left.\left.-H_{\mathrm{r}}(\theta, \phi)\right), D h\right\} \sin \theta \mathrm{d} \theta \mathrm{d} \phi
\end{aligned}
$$

The line shape function, $G$, was assumed to

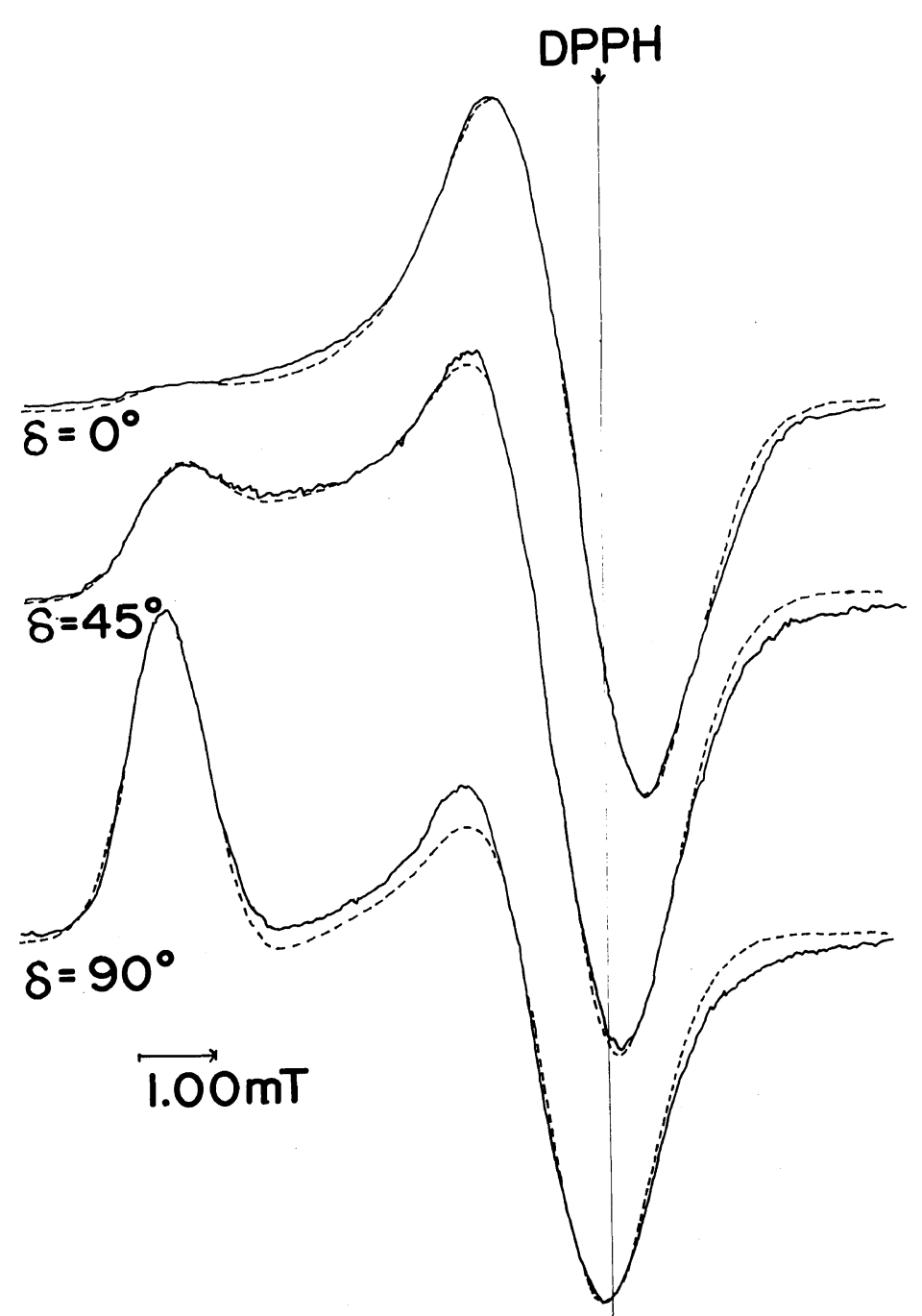

Figure 2. Comparison of experimental spectra of peroxy radicals with simulated spectra for different rotation angles, $\delta$, recorded at $77 \mathrm{~K}$. - experimental; ----, simulated for $f_{\alpha}=0.44$ and $f_{\alpha^{\prime}}=0.10$. 
be Gaussian and line width, $D h$, to vary linearly with the inversion of $g$-value as described in the previous article. ${ }^{3,4}$ Several theoretical spectra were calculated by gradually changing the values of $a$ and $a^{\prime}$. These $f_{\alpha}=\frac{\int_{0}^{\pi / 2}\left[\left(3 \cos ^{2} \alpha-1\right) / 2\right] \exp \left(a \sin ^{2} \alpha\right) \mathrm{d} \alpha}{\int_{0}^{\pi / 2} \exp \left(a \sin ^{2} \alpha\right) \mathrm{d} \alpha}$ spectra were recorded with an $X-Y$ plotter and compared with observed spectra in order to obtain the best fit.

From the determined values of $a$ and $a^{\prime}$, the orientation functions, $f_{\alpha}$ and $f_{\alpha^{\prime}}$ were calculated as follows. ${ }^{1}$

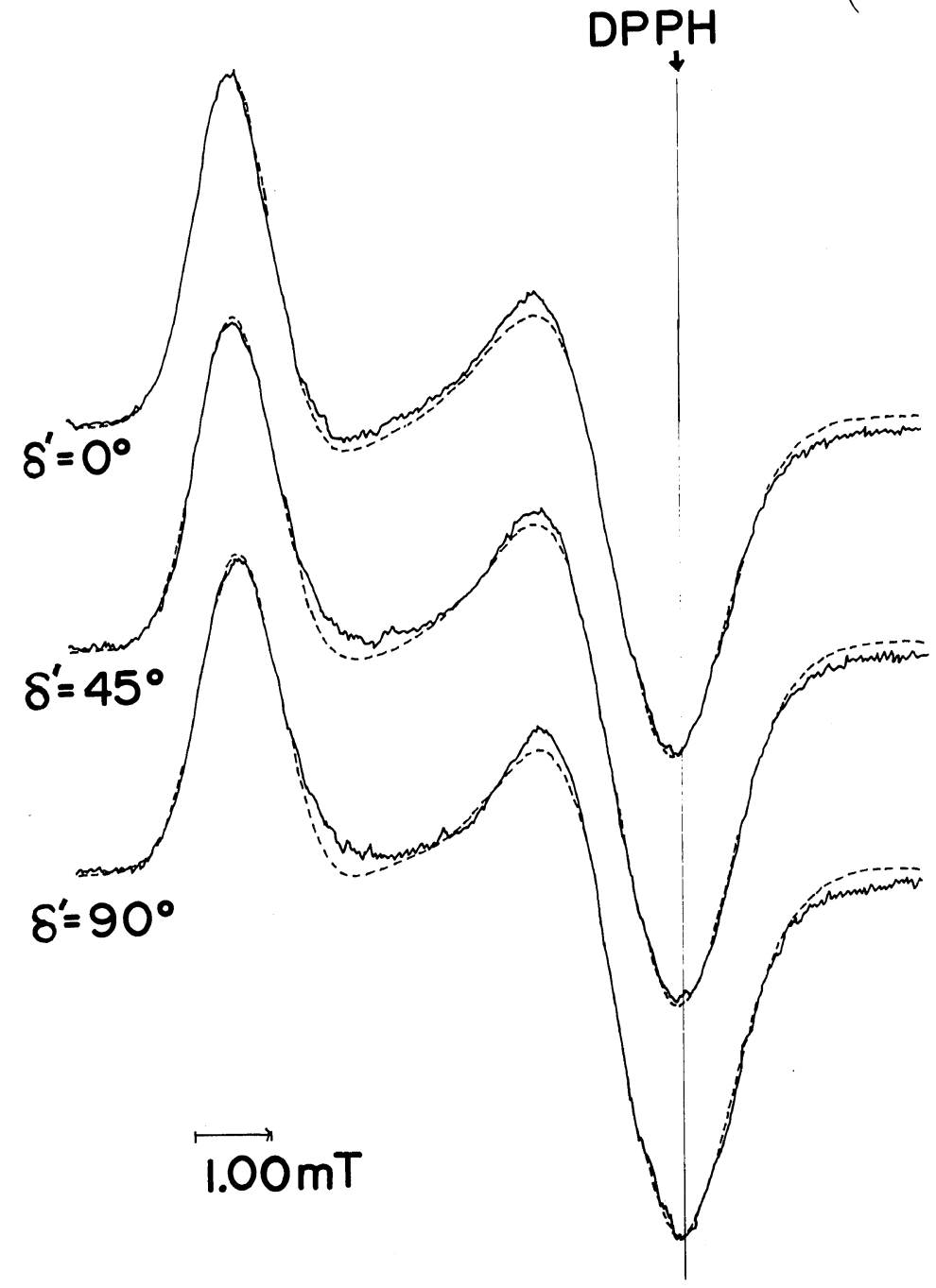

Figure 3. Comparison of experimental spectra of peroxy radicals with simulated spectra for different rotation angles, $\delta^{\prime}$, recorded at $77 \mathrm{~K} ;-$, experimental; - - simulated for $f_{\alpha}=0.47$ and $f_{\alpha^{\prime}}=0.10$. 


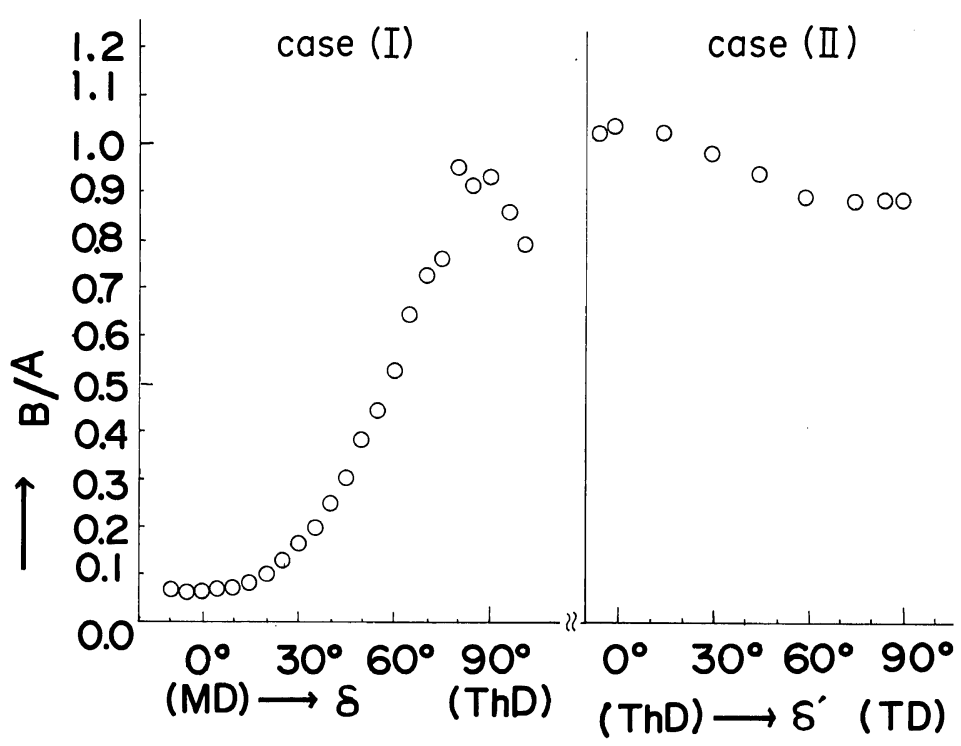

Figure 4. Angular dependence of the shape parameter, B/A. Peak heights of A and B are given in Figure 5.

$f_{\alpha^{\prime}}$ is the degree of orientation of the polymer chain deviating from the film surface. When all polymer chains are oriented parallel to the film surface, $f_{\alpha^{\prime}}=1$.

\section{RESULTS AND DISCUSSION}

Figures 2 and 3 show the angular dependence of the ESR spectra of the oriented PTFE films treated with $\mathrm{C}_{2} \mathrm{H}_{4}$ to eliminate unstable peroxy radicals.

When the sample in configuration (I) was examined, the ESR spectrum showed the remarkable angular dependence as illustrated in Figure 2. For the external magnetic field along the stretching direction $\left(\delta=0^{\circ}\right)$, the intensity of $g_{1}$ was enhanced and that of $g_{2}$ and $g_{3}$ reduced compared to the spectrum obtained for the field perpendicular to the stretching direction $\left(\delta=90^{\circ}\right)$. These finding are consistent with the previous assumption that the direction of $g_{1}$ is parallel to the polymer chain axis, since the polymer chains preferentially oriented along the stretching direction. But for the sample with configuration (II), the angular depen-

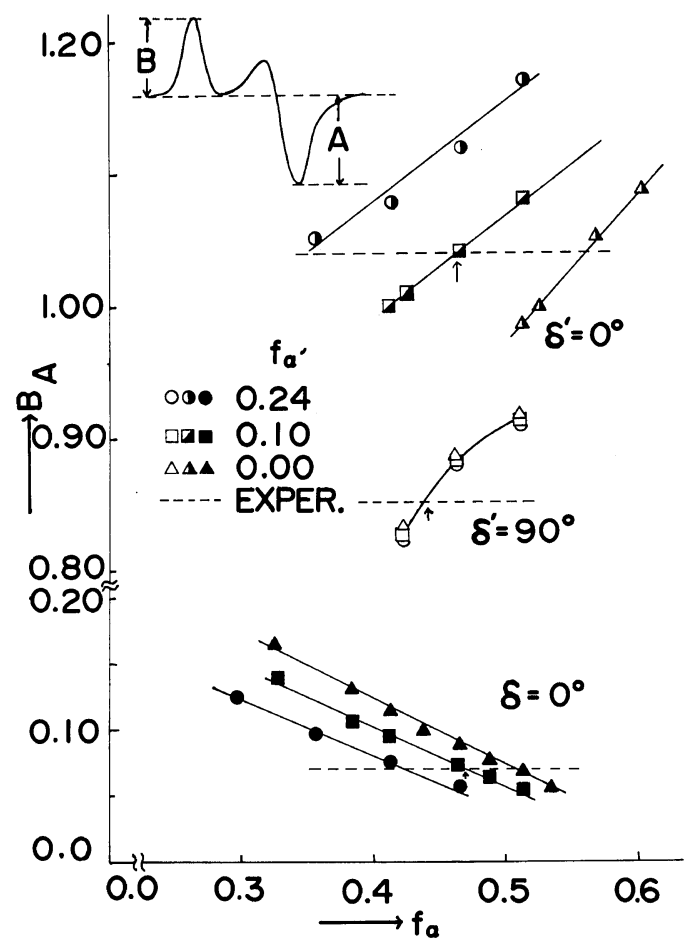

Figure 5. Dependence of the shape parameter, B/A, on the degree of orientation $f_{\alpha}$ for the value, $f_{\alpha^{\prime}}$. 


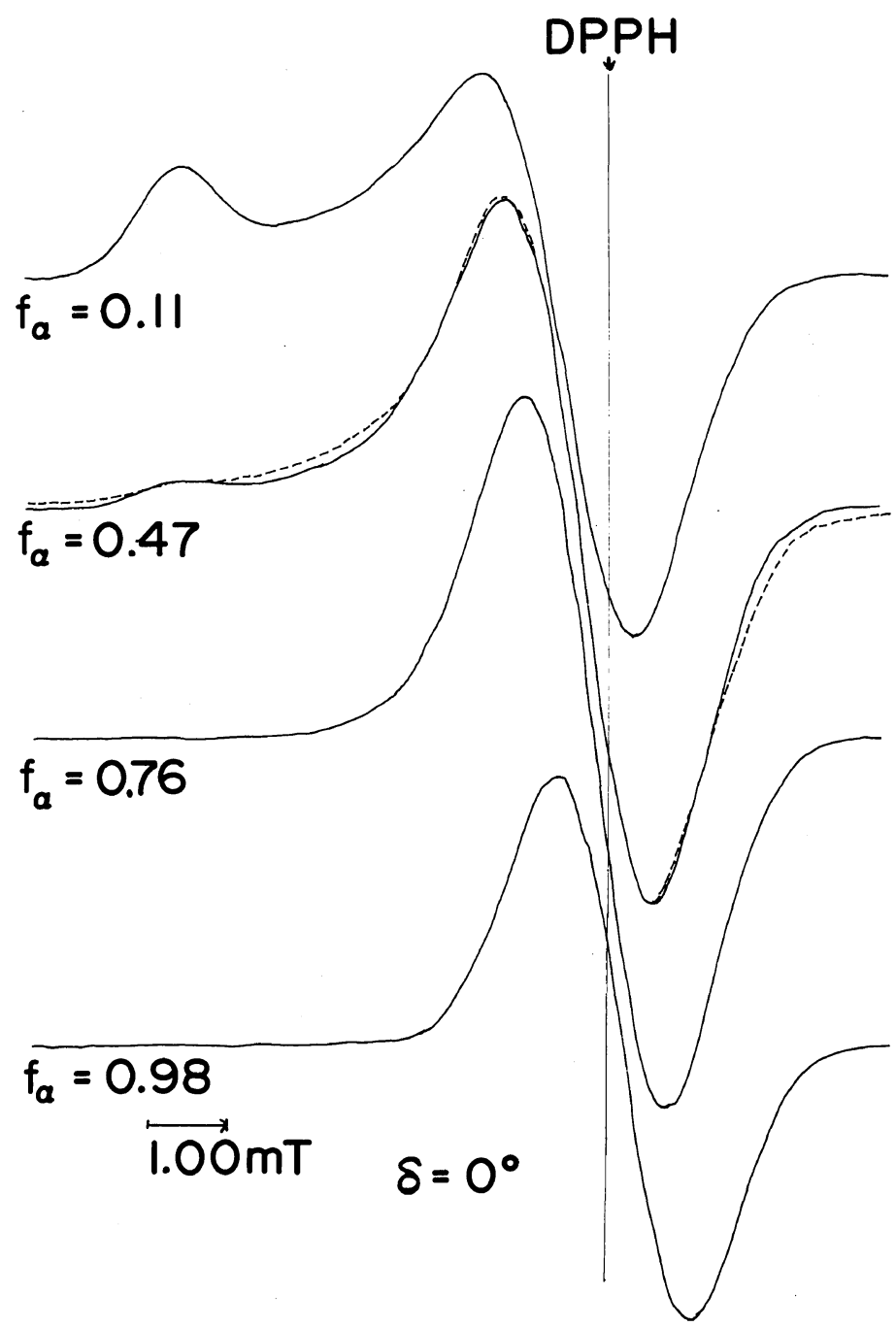

Figure 6. Dependence of ESR spectra of peroxy radicals on the degree of orientation, $f_{\alpha^{\prime}}$ for $f_{\alpha^{\prime}}=0.10$.

dence of the ESR spectrum was reduced more than that of case (I) as evident from Figure 3. For visualization of the angular dependence, the shape parameters, $\mathrm{B} / \mathrm{A}$, were plotted for the angles of rotation, $\delta$ and $\delta^{\prime}$ as shown in Figure 4. The intensities of peaks $\mathrm{A}$ and $\mathrm{B}$ are given in Figure 5.

The angular dependence as represented in Figure 4(II) is not due to the perfect uniaxial orientation of cylindrical symmetry in the stretching direction. The ESR spectrum may possibly be simulated using the two orien- tation functions, $f_{\alpha}$ and $f_{\alpha^{\prime}}$. Figure 5 shows the calculated shape parameters, B/A, of the ESR spectra when of $\delta^{\prime}=0^{\circ}, 90^{\circ}$ and $\delta=0^{\circ}$ for various values of $\dot{f}_{\alpha}$ and $f_{\alpha^{\prime}}$. The shape parameters calculated for $f_{\alpha}(0.43 \sim 0.47)$ and $f_{\alpha^{\prime}}$ (0.10) are consistent with the experimental values for three angles of rotation. The best fitted simulated and experimental spectra are also shown as the dotted and solid lines in Figures 2 and 3 , respectively. The agreement between the simulated and observed spectra was very good for the values of $f_{\alpha}=0.43 \sim 0.47$ and 
$f_{\alpha^{\prime}}=0.10$.

This agreement when $\delta=45^{\circ}$ and $\delta^{\prime}=45^{\circ}$ suggest the estimated orientation functions to be reliable. Figure 6 shows examples of computer simulated spectra generated for different orientation parameters, $f_{\alpha}$ and $a$ constant orientation parameter $f_{\alpha^{\prime}}=0.10$ with $\delta=0^{\circ}$. As expected, Figure 6 shows a very strong dependence of ESR spectrum of partially oriented peroxy radicals on the degree of order.

According to the above data, it may be concluded that ESR spectra of peroxy radicals labeled on the polymer chain are quite sensitive to molecular orientation and ESR is a simple method for determining the orientation to two directions in polymeric materials.

Acknowledgement. The partial support by a Grant-in-Aid for Scientific Research from the Ministry of Education, Science and Culture of Japan is gratefully acknowledged.

\section{REFERENCES}

1. R. J. Samuels, "Structured Polymer Properties," Wiley, New York, N. Y., 1974, p 16, Chapter 2.

2. For example, D. Suryanarayana and L. Kevan, $J$. Phys. Chem., 86, 2042 (1982); D. Suryanarayana, L. Kevan, and S. Schlick, J. Am. Chem. Soc., 104, 668 (1982) and the references cited in these papers.

3. Y. Hori, S. Shimada, and H. Kashiwabara, Polymer, 18, 1143 (1977).

4. Y. Hori, S. Shimada, and H. Kashiwabara, Polymer, 18, 567 (1977).

5. D. Oliver, C. Marachi, and M. Che, J. Chem. Phys., 72, 3348 (1980).

6. M. Iwasaki and Y. Sakai, J. Polym. Sci., 6, 265 (1968).

7. For example, James et al. (a) assumed gaussian distribution, $\quad C \exp \left(a \sin ^{2} \alpha+b \sin ^{4} \alpha\right)$. Workers (a) (d) used different distribution functions. (a) P. G. James and G. R. Luckhurst, Mol. Phys., 19, 489 (1970); (b) L. J. Libertini, C. A. Burle, P. C. Jost, and O. H. Griffith, J. Mag. Res., 15, 460 (1974); (c) H. Blum, J. C. Salerno, and J. S. Leigh, Jr., J. Mag. Res., 36, 385 (1978); (d) J. C. Swartz, B. M. Hoffman, R. J. Krizek, and D. K. Atmatzidis, J. Mag. Res., 36, 259 (1979). 